\title{
CONTACT STRUCTURES ON 3-MANIFOLDS ARE DEFORMATIONS OF FOLIATIONS
}

\author{
JOHN B. ETNYRE
}

\begin{abstract}
In this note we observe, answering a question of Eliashberg and Thurston in [2], that all contact structures on a closed oriented 3 -manifold are $C^{\infty}$-deformations of foliations.
\end{abstract}

\section{Introduction}

In [2], Eliashberg and Thurston proved that foliations could be approximated by contact structures. More precisely they established that any two dimensional $C^{2}$ foliation on a closed oriented 3-manifold $M$, other than the product foliation of $S^{2} \times$ $S^{1}$, can be $C^{0}$-approximated by a positive and negative contact structure. This result brings up the natural question: Is every contact structure on a closed 3-manifold "close" to a foliation? To make sense of "close" we could instead ask, as Eliashberg and Thurston did in [2]: Is every contact structure on a closed 3-manifold a deformation of a foliation? We say a contact structure $\zeta$ is the deformation of a foliation $\xi$ if there is a 1-parameter family of plane fields $\xi_{t}$ such that $\xi_{0}=\xi, \xi_{1}=\zeta$ and $\xi_{t}$ is a contact structure for $t>0$. In this note we show that answer to this last question is indeed yes.

Theorem 1. Every positive and negative contact structure on a closed oriented 3manifold is a $C^{\infty}$-deformation of a $C^{\infty}$-foliation.

The proof of this relies on the connection between open books and contact structures discovered by Giroux [6]. Starting form open books it is not hard to prove this theorem, but it does illuminate the nature of confoliations and the "boundary" of the space of contact structures in the space of plane fields. In the last section we make a few comments concerning the theorem and its proof.

\section{Open books and contact structures}

Recall an open book decomposition of a closed oriented 3-manifold $M$ is a pair $(L, \pi)$ where $L$ is an oriented link in $M$ and $\pi:(M \backslash L) \rightarrow S^{1}$ is a fibration with $\partial\left(\overline{\pi^{-1}(\theta)}\right)=L$ for all $\theta \in S^{1}$. We call $L$ the binding of the open book and the fibers of $\pi$ the pages of the open book. Given an open book we can describe $M \backslash L$ as the mapping cylinder of a diffeomorphism $\psi: \Sigma \rightarrow \Sigma$ of a surface $\Sigma$. Indeed, we can recover $M$ and the open book $(L, \pi)$, up do diffeomorphism, from this data $(\Sigma, \psi)$. We call $\psi$ the monodromy of the open book. For more on open books see [3].

A contact structure $\xi$ is said to be supported by an open book $(L, \pi)$ if there is a contact 1-form $\alpha$ for $\xi$ such that $\alpha(L)>0$ and $d \alpha$ is a volume form when restricted to

Received by the editors August 3, 2006. 
each page of the open book. Thurston and Winkelnkemper, in [7], showed that any open book supports some contact structure and it was observed by Giroux that this contact structure is unique. Moreover, Giroux proved the following correspondence.

Theorem 2 (Giroux, 2002 [6]). If $M$ is a closed oriented 3-manifold then there is a one-to-one correspondence between oriented contact structures on $M$ up to isotopy and open book decompositions of $M$ up to positive stabilization.

It is not important for our arguments what the definition of positive stabilization is, so we do not define it here. Our main use of this theorem will be the facts that (1) all contact structures are supported by open books and (2) the supported contact structure is unique up to isotopy.

\section{Proof of Theorem 1}

To prove Theorem 1 we start with a positive contact structure $\zeta$ on a closed oriented 3 -manifold $M$ and then choose some open book $(L, \pi)$ for $M$ that supports $\zeta$. (The proof for negative contact structures is similar. The details are left as an easy exercise for the reader.) We will then construct a foliation on $M$ associated to this open book and show that it can be perturbed into a contact structure that is also supported by $(L, \pi)$. Thus the perturbed contact structures will have to be isotopic to $\zeta$, confirming the theorem. We will denote the page and monodromy of the open book by $(\Sigma, \psi)$.

One can construct an obvious foliation with Reeb components by replacing neighborhoods of the binding with Reeb components and then "spinning" the pages of the open book so they limit to the Reeb components. We describe the procedure more carefully. Let $N$ be a neighborhood of one component of the binding. Choose coordinates $(r, \theta, \phi)$, so that the pages of the open book intersect $N$ correspond to constant $\theta$ annuli and the binding corresponds to $r=0$. Moreover assume $N=\{(r, \theta, \phi) \mid r \leq 1+2 \epsilon\}$ for some small fixed $\epsilon$. We now choose two functions $\lambda(r)$ and $\delta(r)$ so that $\lambda$ is zero on $\left[0, \frac{1}{3}\right]$, one for $r \geq 1$, and strictly increasing on $\left[\frac{1}{3}, 1\right]$, and $\delta$ is zero on $[0,1]$, one for $r \geq 1+\epsilon$ and strictly increasing on $[1,1+\epsilon]$. Now set

$$
\alpha= \begin{cases}\lambda(r) d r+(1-\lambda(r)) d \phi & \text { for } r \leq 1 \\ \delta(r) d \theta+(1-\delta(r)) d r & \text { for } r>1\end{cases}
$$

One may easily check that $\alpha \wedge d \alpha=0$ so $\xi=\operatorname{ker} \alpha$ gives a foliation of $N$. The subset $N_{1}=\{(r, \theta, \phi) \mid r \leq 1\}$ of $N$ is a Reeb component. (We will denote by $N_{a}$ the set $\{(r, \theta, \phi) \mid r \leq a\}$.) Note we can choose $\lambda(r)$ and $\delta(r)$ so that $\alpha$ defines a $C^{\infty}$-foliation on $N$. It is clear that in $[1+\epsilon, 1+2 \epsilon] \times T^{2}$ the foliation is by constant $\theta$ annuli. Thus this foliation can be extended by the pages of the open book on $M \backslash N$ to a foliation of all of $M$. (Of course, we insert this standard model with a Reeb component for each component of the binding $L$.) If we set $d z$ to the pull back of the coordinate $\vartheta$ on $S^{1}$ by the fibration $\pi: M \backslash L \rightarrow S^{1}$ then we can extend $\alpha$ by $d z$ to get a 1 -form defining our foliation on all of $M$.

We now show how to perturb this foliation in to a contact structure supported by the open book $(L, \pi)$. Again, we start by concentrating on the neighborhood $N$ of a component of the binding $L$. Here we set

$$
\alpha_{t}=\alpha+t\left(r^{2} d \theta+(1+f(r)) d \phi\right)
$$


where $f: N \rightarrow \mathbb{R}$ is a function that is strictly decreasing, $f(0)=0, f(r)>-1$ for all $r$ and $f(r)<-1+\iota$ for all $r>1$ and $\iota$ some small number. Now we compute

$$
d \alpha_{t}= \begin{cases}\left(t f^{\prime}(r)-\lambda^{\prime}(r)\right) d r \wedge d \phi+t 2 r d r \wedge d \theta & \text { for } r \leq 1 \\ \left(t 2 r+\delta^{\prime}(r)\right) d r \wedge d \theta+t f^{\prime}(r) d r \wedge d \phi & \text { for } r>1\end{cases}
$$

Thus we have

$$
\begin{aligned}
& \alpha \wedge d \alpha_{t}= \\
& \begin{cases}\operatorname{tr}\left(2[(1-\lambda(r))+t(1+f(r))]-r\left(t f^{\prime}(r)-\lambda^{\prime}(r)\right)\right) d r \wedge d \theta \wedge d \phi & \text { for } r \leq 1, \\
t\left(-f^{\prime}(r)\left(\delta(r)+t r^{2}\right)+(1+f(r))\left(t 2 r+\delta^{\prime}(r)\right)\right) d r \wedge d \theta \wedge d \phi & \text { for } r>1 .\end{cases}
\end{aligned}
$$

From this formula, it may easily be checked that $\alpha_{t}$ is a contact from on $N$ for $t>0$.

We would like to patch this into a family of 1 -forms on $M \backslash N_{1+\epsilon}$. To this end we briefly recall the Thurston-Winkelnkemper construction of contact forms on open books, [7]. We begin by thinking of $M \backslash N_{1+\epsilon}$ as the mapping cylinder of $\psi: \Sigma \rightarrow \Sigma$, the monodromy of the open book. That is

$$
M \backslash N^{\prime}=\Sigma \times[0,1] / \sim,
$$

where $(\psi(x), 0) \sim(x, 1)$, and the coordinate on the $[0,1]$ factor is $z$. We then find a 1-parameter family of 1 -forms $\lambda_{z}$ on $\Sigma$ so that $d \lambda_{z}$ is a volume form on $\Sigma$ for all $z$ and each $\lambda_{z}=(1+\epsilon+s) d \theta$ near each boundary component of $\Sigma$, where we use "polar" coordinates $(s, \theta)$ near the boundary component and the boundary corresponds to $s=0$ and $s$ is increasing into $\Sigma$. Moreover, the $\lambda_{z}$ are chosen so that they descend to give a form on $M \backslash N_{1+\epsilon}$. Note the 1-form $d z$, from the second paragraph of this section, corresponds to $d z$ in these coordinates. The 1 -form $\beta_{t}=d z+t \lambda_{z}$ will be a contact 1-form on $M \backslash N_{1+\epsilon}$ for small $t>0$.

To patch the two 1-forms together we consider the region $A=\overline{N \backslash N_{1+\epsilon}}$. We use the above coordinates on $N$ as coordinates on $A$. Near the boundary of $M \backslash N$ in $A$ the contact 1 -form is $\beta_{t}=d z+t(1+\epsilon+s) d \theta$. We use the map $\Psi(r, \theta, \phi)=(r-1-\epsilon,-\phi, \theta)$ to map $A \subset N$ to a neighborhood of the boundary of $M \backslash N_{1+\epsilon}$. It is easy to check that this map is orientation preserving and when $N$ is glued to $M \backslash N_{1+\epsilon}$ using this map we recover $M$. Pulling $\beta_{t}$ back to $A$ using this map we get $\Psi^{*} \beta_{t}=-t r d \phi+d \theta$. We think of this form as defined only near $T_{1+2 \epsilon}=\partial N_{1+2 \epsilon}$ in $A$. Similarly $\alpha_{t}=$ $\left(1+t r^{2}\right) d \theta+t(1+f(r)) d \phi$ is a form defined near $T_{1+\epsilon}=\partial N_{1+\epsilon}$ in $A$. In order to interpolate between these two forms we consider forms on $A$ of the type

$$
\gamma=g(r) d \phi+h(r) d \theta
$$

This will be a contact form if and only if $g(r) h^{\prime}(r)-h(r) g^{\prime}(r) \neq 0$, that is if we think of $(g(r), f(r))$ as parameterizing a curve in $\mathbb{R}^{2}$ then the position vector and velocity vector can never be co-linear. If we take $g(r)$ and $h(r)$ to be defined by $\Psi^{*} \beta_{t}$ and $\alpha_{t}$ near the boundary of $A$ then we can clearly extend $g(r)$ and $h(r)$ to all of $A$ so that we have a contact form on $A$. Moreover, it is easy to check that we can choose $g(r)$ so that $g^{\prime}(r)<0$ in $A$.

Let $\alpha_{t}$ be the 1 -from on $M$ that equals $\alpha_{t}$ on $N_{1+\epsilon}, \beta_{t}$ on $M \backslash N$ and the from $g(r) d z+h(r) d \theta$ just constructed on $A$. This gives a well defined form for all $t \geq 0$. Moreover, $\alpha_{0}$ is the form $\alpha$ above that defines the foliation $\xi$ and for small $t>0$, 
$\alpha_{t}$ defines a contact structure $\xi_{t}=\operatorname{ker} \alpha_{t}$. Thus the contact structure $\xi_{t}$ is clearly a deformation of the foliation $\xi$.

We are left to show that $\xi_{t}$ is supported by the open book $(L, \pi)$. For this we must see that $\alpha_{t}(L)>0$ and $\left.d \alpha_{t}\right|_{\text {page }}$ is a volume form on $\Sigma$. A component of $L$ corresponds to $r=0$ in $N$ and its positively oriented tangent vector is given by $\frac{\partial}{\partial \phi}$. So $\alpha_{t}\left(\frac{\partial}{\partial \phi}\right)=d \phi\left(\frac{\partial}{\partial \phi}\right)=1>0$. To check the second condition we consider the four regions $N_{1}, N_{1+\epsilon} \backslash N_{1}, A$ and $M \backslash N$. On $N_{1}$ the pages of the open book correspond to constant $\theta$ annuli. The form $d \alpha_{t}$ restricted to this annulus is $\left(t f^{\prime}(r)-\lambda^{\prime}(r)\right) d r \wedge d \phi$. This form is never zero and the coefficient is always negative in $N_{1}$, but the orientation on the annulus that allows for $L$ to be properly oriented corresponds to the form $d \phi \wedge d r$. So $d \alpha_{t}$ is a properly oriented non-zero 2 -form on the pages in $N_{1}$. Now on $N_{1+\epsilon} \backslash N_{1}$ the pages are still constant $\theta$ annuli and the 1 -form restricts to $t f^{\prime}(r) d r \wedge d \phi$ on these. Thus $\alpha_{t}$ is compatible withe the pages in this region. On $A$ the pages are again constant $\theta$ annuli, so the form restricted to this is $g^{\prime}(r) d r \wedge d \phi$. By the choice of $g$ above this is a properly oriented non-zero 2 -form on the pages in $A$. Finally in $M \backslash N$ it is clear $d \beta_{t}$ is a properly oriented non-zero 2 -form on the pages by construction (see $[7])$.

\section{Comments}

In [2] it was shown that any confoliation is isotopic through confoliations to an overtwisted contact structure. Using Theorem 1 we can see other interesting facts concerning isotopies of confoliations. Eliashberg and Thurston generalized the notion of tightness to confoliations. They say a confoliation $\xi$ is tight if for every disk $D$ with $\partial D$ tangent to $\xi$, but $D$ itself transverse to $\xi$ near $\partial D$, there is a disk $D^{\prime}$ with the same boundary as $D$ that is tangent to $\xi$ everywhere and satisfies $\left\langle e(\xi),\left[D \cup D^{\prime}\right]\right\rangle=0$, where $e(\xi)$ is the Euler class of the plane field $\xi$. If $\xi$ is a contact structure this agrees with the usual definition of tight. If $\xi$ is a foliation the is equivalent to the foliation being Reebless. Eliashberg and Thurston claimed that the perturbation of a tight foliation is a tight contact structure (and in fact tight when pulled back to the universal cover). While there seems to be a gap in their argument, see [1], the result is true, see [4]. We have the immediate corollary of Theorem 1.

Corollary 3. If $\xi$ is a tight foliation then we can isotop $\xi$ through tight confoliations to a foliation with Reeb components. In particular, a taut foliation is isotopic to a foliation with Reeb components via an isotopy through tight confoliations.

In the definition of the foliation in Section 3 the reader may verify that the leaves of the foliations coming from the pages of the open book spiraled towards the Reeb component in a clockwise manner (as seen in a constant $\phi$ slice). We could have considered a foliation that spiraled in the opposite direction or changed the direction of the spiraling in the Reeb component itself. Making either of these changes (but not both) would have resulted in a foliation that perturbs to an overtwisted contact structure. (To see this take a meridional disk in $N$ of radius slightly larger than one. This will give a transverse unknot in the contact structure that violates the Bennequin inequality.) So if the open book supported a tight contact structure then this perturbed contact structure could not be supported by the open book. More 
generally, it is not too hard to see that the perturbed contact structure will never be supported by the given open book.

Finally we observe that if a contact structure is virtually overtwisted (i.e. becomes overtwisted when pulled back to a finite cover) then any foliation of which it is a perturbation must contain Reeb components, since Reeblees foliations are tight and perturb to tight contact structures. Thus the foliations constructed in the proof Theorem 1 are as good as can be expected for most contact structures. However, if a contact structure is universally tight (i.e. its pull back to the universal cover is tight) then one may hope for better. In particular, we end with the following questions:

Question 4. If $M$ has infinite fundamental group then is every universally tight contact structure the deformation of a Reebless foliation?

Question 5. If $M$ is atoroidal and has infinite fundamental group then is every universally tight contact structure the deformation of a taut foliation?

The condition on the fundamental group is necessary since a theorem of Novikov says that if the fundamental group is finite then there must be a Reeb component in the foliation. The atoroidal assumption is necessary because Ghiggini [5] has constructed universally tight contact structures that cannot be weakly fillable and hence cannot be perturbations of taut foliations.

\section{Acknowledgements}

The author thanks Baptiste Chantraine, Paolo Ghiggini and the referee for helpful comments on the first version of the paper. The work in this paper was supported in part by NSF CAREER Grant (DMS-0239600) and FRG-0244663.

\section{References}

[1] V. Colin, Structures de contact tendues sur les variétés toroïdales et approximation de feuilletages sans composante de Reeb, Topology 41 (2002), no. 5, 1017-1029.

[2] Y. M. Eliashberg and W. P. Thurston, Confoliations, Vol. 13 of University Lecture Series, American Mathematical Society, Providence, RI (1998), ISBN 0-8218-0776-5.

[3] J. B. Etnyre, Lectures on open book decompositions and contact structures. Proceedings of the "Floer Homology, Gauge Theory, and Low Dimensional Topology", 103-141, Clay Math. Proc, 5, Amer. Math. Soc. 2006.

[4] - Perturbations of Reebless Foliations (2006). In preparation.

[5] P. Ghiggini, Infinitely many universally tight contact manifolds with trivial Ozsváth-Sazbó contact invariants. Geom. Topol. 10, (2006), 335-357 (electronic).

[6] E. Giroux, Géométrie de contact: de la dimension trois vers les dimensions supérieures, in Proceedings of the International Congress of Mathematicians, Vol. II (Beijing, 2002), 405-414, Higher Ed. Press, Beijing (2002).

[7] W. P. Thurston and H. E. Winkelnkemper, On the existence of contact forms, Proc. Amer. Math. Soc. 52 (1975) 345-347.

School of Mathematics, Georgia Institute of Technology, 686 Cherry Street, Atlanta, GA $30332-0160$

E-mail address: etnyre@math.gatech.edu

$U R L:$ http://www.math.gatech.edu/ etnyre 